medication. A small proportion of individuals switched shortly to SB4 and then later to other medication. $5.7 \%$ of former etanercept users switched to other medication at the time of Switch date. For details, see Figure 1.

Many patients switched back and forth several times, also using other medication at short time intervals. The method of distribution (pen versus injection) was associated with higher odds for being switched. Patients using etanercept pen were more likely to switch to biosimilar etanercept after BE (Figure 2).

For patients who switched to SB4 the 18 months cumulative incidence of drug survival was $72 \%$; $95 \% \mathrm{Cl}[65-79] \%$. At 2 years follow-up the drug survival in switchers will be matched with historic drug survival rate of etanercept estimated using data from NorPD.

Conclusion: The Norwegian Prescription Database is a useful tool that enables to monitor use, changes and trends in use of selected drugs. A longer follow-up is warranted in order to describe drug survival after switch from etanercept to biosimilar etanercept. However, close collaboration between pharmacists, clinicians and statisticians is warranted

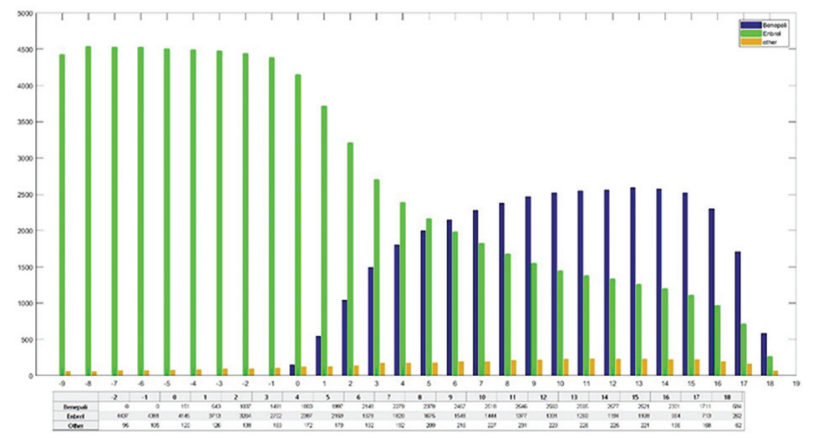

Figure 1

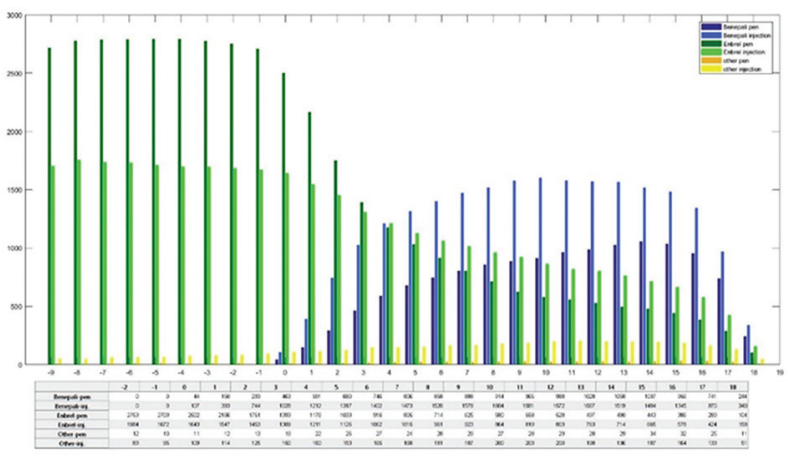

Figure 2

Disclosure of Interests: Milada Cvancarova Småstuen Grant/research support from: I have received a Research grant from Pfizer, Randeep Madla Employee of: Pfizer, still employed, Oddvar Solli Employee of: Pfizer, still employed by Pfizer, Erik Hjelvin Employee of: Pfizer, still employed by Pfizer

DOI: 10.1136/annrheumdis-2019-eular.5799

\section{OP0312 VARIABILITY IN BIOLOGIC PRESCRIPTION PATTERNS FOR RHEUMATOID ARTHRITIS PATIENTS IN THE AMERICAN COLLEGE OF RHEUMATOLOGY INFORMATICS SYSTEM FOR EFFECTIVENESS (RISE) REGISTRY}

Douglas White $^{1}$, Michael Evans ${ }^{2}$, Tracy Johansson $^{3}$, Rachel Myslinski ${ }^{3}$, Jinoos Yazdany ${ }^{2}$, Gabriela Schmajuk ${ }^{2,4}$. 'Tundersen Health System, Onalaska, United States of America; ${ }^{2}$ University of California San Francisco, San Francisco, United States of America; ${ }^{3}$ American College of Rheumatology, Atlanta, United States of America; ${ }^{4}$ Veterans Affairs Medical Center, San Francisco, United States of America

Background: Biologics account for a substantial portion of drug spending in rheumatoid arthritis (RA). Variability in the U.S. in biologic prescribing patterns (and therefore variability in cost of care), and factors that correlate with that variability, remain largely undefined.
Objectives: We used data from the RISE registry to perform a cross-sectional analysis among U.S. rheumatologists of prescription patterns for biologic DMARDs and tofacitinib and their relationship to RA disease activity.

Methods: RISE is a U.S. registry that passively collects data on all patients seen by participating practices, thereby reducing selection bias present in singleinsurer claims databases. As of December 2017, RISE held validated data from 1.257 providers in 236 practices, representing an estimated $25 \%$ of the U.S. clinical rheumatology workforce. We identified patients with available demographic and disease activity information who were assigned $\geq 2$ codes for $R A \geq 30$ days apart between January and December 2017. Practices with $<20$ RA patients (15/ 104 practices providing all necessary data) were excluded. We tallied the proportion of patients in each practice prescribed a TNF inhibitor, abatacept, rituximab tocilizumab, or tofacitinib at least once during 2017. Patients prescribed $>1$ of these drugs were assigned to the first drug prescribed and therefore counted only once. We used a hierarchical linear model to predict the probability of receiving a prescription for a biologic based on the patient's most recent disease activity score (moderate or high disease activity vs. low disease activity or remission) and age from 2016, accounting for clustering by practice.

Results: We analyzed 53.850 patients from 104 practices. Overall, 29\% of patients were prescribed a biologic DMARD or tofacitinib in 2017. TNF inhibitors were most commonly prescribed, followed by abatacept (4.5\% of patients), tofacitinib $(4.2 \%)$, tocilizumab (3.4\%), and rituximab (2.5\%). We found significant variation within practices in the proportion of patients prescribed any of these drugs (range $0 \%-100 \%$ ). In the adjusted analysis, we found that patients with higher disease activity in 2016 were more likely to receive biologics in 2017 (OR 1.56, 95\% $\mathrm{Cl}(1.51,1.62))$. Within a practice, as shown in the figure, the risk-adjusted likelihood of receiving a biologic prescription still showed significant variation (between $0 \%-83 \%$ of patients in each practice received a biologic; model c-statistic 0.61 ).

Figure. Proportion of RA patients prescribed biologics or tofacitinib, aggregated by practice and adjusted for disease activity and age, for practices with at least 20 patients with RA, between January and December 2017.

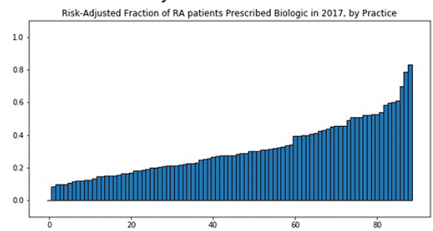

Conclusion: In this large sample of U.S. rheumatology practices, higher RA disease activity correlated with the likelihood that a patient would receive a prescription for a biologic, but did not account for all of the variability in biologic prescription patterns. These results suggest that there may be other factors in addition to RA disease activity that account for practice-to-practice variability in biologic prescription patterns. Disclaimer: These data were collected from the ACR'S RISE Registry; however, the views expressed represent those of the authors, not necessarily those of the ACR. Disclosure of Interests: Douglas White Grant/research support from: Abbott 2006-2009, Pfizer 2017-2019 (unrelated to this work), Consultant for: Crescendo advisory board 2013-2014, Employee of: Genomyx (Genentech) 1991, Speakers bureau: None since 2008, Michael Evans: None declared, Tracy Johansson: None declared, Rachel Myslinski: None declared, Jinoos Yazdany Grant research support from: Pfizer, Consultant for: AstraZeneca, Gabriela Schmajuk Grant/research support from: Investigator initiated award from Pfizer from 2015 2018, unrelated to this work

DOI: 10.1136/annrheumdis-2019-eular.4610

\section{OP0313 \\ COST-EFFECTIVENESS OF A JAK1/JAK2-INHIBITOR VS. A BIOLOGIC DISEASE-MODIFYING ANTIRHEUMATIC DRUG IN A TREAT-TO-TARGET STRATEGY FOR RHEUMATOID ARTHRITIS}

Celine van de Laar ${ }^{1}$, Martijn Oude Voshaar ${ }^{1}$, Walid Fakhouri ${ }^{2}$, Liliana ZarembaPechmann $^{2}$, Francesco de Leonardis ${ }^{2}$, Inmaculada De La Torre ${ }^{2}$, Mart van de Laar ${ }^{1,3}$. ${ }^{1}$ Transparency in Healthcare, Hengelo, Netherlands; ${ }^{2}$ Eli Lilly and Company, Indianapolis, United States of America; ${ }^{3}$ Arthritis Center Twente, Enschede, Netherlands

Background: Treating Rheumatoid Arthritis (RA) to an a priori defined disease activity target (T2T) is recommended in EULAR guidelines. This involves a stepup approach in which it is first attempted to achieve the target with a combination of conventional synthetic (cs) DMARDs. Baricitinib is a JAK1/JAK2-inhibitor approved for treatment of patients suffering from RA. EULAR and ACR guidelines currently position JAK1/JAK2-inhibitors and bDMARDs at the same level in the therapeutic treatment sequence for csDMARD Inadequate Responders (IR) Cost-effectiveness assessment of different T2T strategies, especially ones 
including new treatments, and integrating health economic considerations is of importance to the decision-making process as it incorporates the societal perspective in the longer run

Objectives: To compare the cost-effectiveness of the previously evaluated DREAM T2T strategy csDMARD combination therapy (csDMARDs) $\rightarrow$ first bDMARD (adalimumab) $\rightarrow$ next bDMARD (TNFi/non-TNFi) with the comparable strategy in which baricitinib is placed instead of adalimumab in csDMARDs IR using a Markov model. All analyses were performed from the societal perspective. Costs and effects over five years were compared between the two strategies to provide insight into the differences in economic considerations between treating patients with strategies including either JAK1/JAK2-inhibitor (baricitinib) or $1^{\text {st }}$ bDMARD (adalimumab).

Methods: A Monte Carlo microsimulation model was developed to conduct costutility analysis from the societal perspective over 5 years. Health states were based on the DAS28-ESR categories. Effectiveness of baricitinib was retrieved from clinical trials (1) and corrected for differences between RCT and real-world setting. Effectiveness of all other treatments, health state utilities, medical costs, and productivity loss were retrieved from DREAM cohorts (2) and the Dutch Institute for Health. Annual discount rates of $1.5 \%$ for utility and $4 \%$ for costs were used, as advised in Dutch guidelines. All analyses were run using probabilistic sensitivity analysis (PSA) to incorporate uncertainty around all parameters and assess result robustness.

Results: PSA results showed the baricitinib strategy yielded lower costs and higher utility over a 5-year period and is cost-effective and dominant over the DREAM T2T strategy. Scenario analyses showed the baricitinib strategy to be cost-effective in both the moderate and severe at baseline RA populations analysed together and separately.

Table 1. Probabilistic Sensitivity Analysis Results

\begin{tabular}{|c|c|c|c|c|c|}
\hline & Costs $(€)(\mathrm{Cl})$ & $\begin{array}{c}\text { Effects (QALY) } \\
(\mathrm{Cl})\end{array}$ & $\Delta \mathrm{C}$ & $\Delta \mathrm{E}$ & ICER \\
\hline DREAM T2T & $\begin{array}{c}14288.36 \\
(10104.68- \\
19053.39)\end{array}$ & $\begin{array}{c}3.5607 \\
(2.4028-4.7236)\end{array}$ & & & \\
\hline $\begin{array}{l}\text { Baricitinib } \\
\text { strategy }\end{array}$ & $\begin{array}{c}13430.57 \\
(9475.10-17848.92)\end{array}$ & $\begin{array}{c}3.5643 \\
(2.4042-4.7315)\end{array}$ & -857.79 & 0.0036 & Dominant \\
\hline
\end{tabular}

$\mathrm{Cl}=95 \%$ Confidence Interval, QALY $=$ Quality-Adjusted Life Year, $\triangle \mathrm{C}=$ Cost difference, $\Delta E=$ Effect Difference, ICER = Incremental Cost-Effectiveness Ratio Conclusion: Results suggest the use of a JAK1/JAK2-inhibitor (baricitinib) instead of a $1^{\text {st }}$ bDMARD (adalimumab) in a T2T strategy is cost-effective in treating RA patients.

\section{REFERENCES:}

[1] Taylor, P. C., Keystone, E. C., van der Heijde, D., Weinblatt, M. E., del Carmen Morales, L., ... \& Arora, V. (2017). Baricitinib versus placebo or adalimumab in rheumatoid arthritis. New England Journal of Medicine, 376 (7), 652-662

[2] M., Versteeg, G. A., Vonkeman, H. E., Ten Klooster, P. M., Kuper, H. H., ... \& van de Laar, M. A. (2016). Initial combination therapy versus step-up therapy in treatment to the target of remission in daily cl. Steunebrink, L. inical practice in early rheumatoid arthritis patients: results from the DREAM registry. Arthritis Research \& Therapy, 18(1), 60.

Disclosure of Interests: Celine van de Laar: None declared, Martijn Oude Voshaar: None declared, WALID FAKHOURI Shareholder of: Eli Lilly and Company, Employee of: Eli Lilly and Company, Liliana Zaremba-Pechmann Shareholder of: Eli Lilly and Company, Employee of: Eli Lilly and Company, Francesco de Leonardis Shareholder of: Eli Lilly and Company, Employee of: Eli Lilly and Company, Inmaculada De La Torre Shareholder of: Eli Lilly and Company, Employee of: Eli Lilly and Company, Mart van de Laar Grant/research support from: Eli Lilly, Pfizer, Merck, AbbVie and Janssen Cilag, Consultant for: Sanofi Genzym, Eli Lilly, Pfizer, Merck, Abbvie and Janssen Cilag, Speakers bureau: Eli Lilly, Pfizer and Janssen Cilag

DOI: 10.1136/annrheumdis-2019-eular.906
FRIDAY, 14 JUNE 2019

\section{Primary and secondary fibromyalgia; are they different?}

\begin{tabular}{|l|l}
\hline OP0314 & LONG TERM TRAJECTORIES OF CHRONIC \\
WIDESPREAD PAIN: A 21-YEAR PROSPECTIVE \\
COHORT LATENT CLASS ANALYSIS
\end{tabular}

Katarina Aili ${ }^{1,2,3}$, Paul Campbell ${ }^{4,5}$, Zoe A Michaleff ${ }^{4}$, Victoria Y. Strauss ${ }^{6}$, Kelvin Jordan ${ }^{4,7}$, Ann Bremander ${ }^{1,8}$, Peter Croft ${ }^{4,7}$, Stefan Bergman ${ }^{1,9}$. ${ }^{1}$ RandD Spenshult, Halmstad, Sweden; ${ }^{2}$ Halmstad University, School of Health and Welfare, Halmstad, Sweden; ${ }^{3}$ Karolinska Institutet, Institute of Environmental Medicine, Stockholm, Sweden; ${ }^{4}$ Keele University, Research Institute for Primary Care and Health Sciences, Keele, United Kingdom; ${ }^{5}$ Midlands Partnership NHS Foundation Trust, Stafford, United Kingdom; ${ }^{6}$ University of Oxford, CSM, NDORMS, Oxford, United Kingdom; ${ }^{7}$ Keele University, Centre for Prognosis Research, Keele, United Kingdom; ${ }^{8}$ University of Southern Denmark, Department of Regional Health Research, Odense, Denmark; ${ }^{9}$ University of Gothenburg, Institute of Medicine, Gothenburg, Sweden

Background: Chronic widespread pain (CWP) is common (population prevalence of approximately $10 \%$ ) and has a significant impact on the individual, healthcare, and society. Currently little is known about the actual course of CWP over time, in particular the pathways to the development and maintenance of CWP. One useful way to understand these pathways is to identify common clusters of people who share pain trajectories. Such information is clinically useful to identify factors that predict development, persistence, and resolution of CWP.

Objectives: To identify different longitudinal pain trajectories over a period of 21 years.

Methods: A 21-year longitudinal open-population cohort of $n=1858$ adults (aged 20-74) who completed surveys relating to their pain status in at least three of the five time points 1995, 1998, 2003, 2007, and 2016. Pain status (presence of persistent pain) was ascertained from a report of painful regions (0-18) on a pain mannequin and categorised into: NCP (No chronic pain), CRP (Chronic regional pain) and CWP (chronic widespread pain). Latent Class Growth Analysis (LCGA) was carried out based on these categories. Participants were assigned to a trajectory cluster where the posterior probability was the highest. Model fit was assessed by statistical indices and clinical interpretations of clusters.

Results: LCGA identified five clusters describing different pathways of NCP, CRP and CWP over the 21 years. The cluster "Persistent NCP" was the most common pathway $(n=1052,57 \%)$ representing those with no chronic pain over the whole time period. The "Persistent CRP or Migration from CRP to NCP" cluster included 411 individuals (22\%) representing a group with stable or improving regional pain. In the groups who were shown to increase pain status, the "Migration from NCP to CRP or CWP" cluster included 92 individuals (5\%), and there were 184 individuals (10\%) in the cluster "Migration from CRP to CWP" representing a group with regional pain who developed CWP. The final cluster "Persistent CWP" included 119 individuals (6\%) representing those with stable CWP throughout the time period. Figure 1 presents the mean number of pain sites over time by cluster.

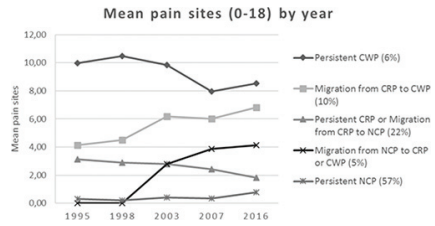

Figure 1. Mean pain sites for each cluster for each point in time.

Conclusion: This study showed that whilst half of adults report no chronic pain over 21 years, a substantial proportion develop CWP or have persistent CWP over this time period. Whilst a common trajectory was movement from chronic regional pain to no chronic pain, a pattern of improving CWP was not seen suggesting this is an uncommon trajectory. This is the first study to show long-term trajectories for CWP, and further work is now required to understand factors that may identify individuals at risk of worsening pain status and factors that might promote improvement. These identified pathways of chronic pain over a lifespan improve the understanding of long-term development of chronic pain and chronic widespread pain.

Disclosure of Interests: None declared

DOI: 10.1136/annrheumdis-2019-eular.2993 